\title{
Use of Oxidative Stress Markers in Diagnosis and Evolution of Age-Related Macular Degeneration
}

\begin{abstract}
RALUCA EUGENIA IORGA ${ }^{1 *}$, RAZVANA SORINA MUNTEANU DANULESCU², MANUELA OZTURK³, DANUT COSTIN ${ }^{1,3}$
IProf. Dr. Nicolae Oblu Emergency Hospital, $2^{\text {nd }}$ Ophthalmology Clinic, 2 Ateneului Str., 700309, Iasi, Romania

${ }^{2}$ Grigore T. Popa University of Medicine and Pharmacy, Department of Gastroenterology, 6 Universitatii Str., 700115, Iasi, Romania ${ }^{3}$ Grigore T. Popa University of Medicine and Pharmacy, Department of Ophthalmology, 6 Universitatii Str., 700115, Iasi, Romania

Age-related macular degeneration (AMD) is the most common cause of blindness in the elderly. Were monitored the evolution of oxidative stress markers before and after treatment. This is a case-control study, which included 59 patients diagnosed with AMD and 64 healthy controls. According to the AREDS classification, the patients were divided into two classes, moderate and severe form AMD and were treated with antioxidants, neurotrophic drugs, intravitreal injections with antiangiogenic factors. We followed the patients over a 12-month period and we evaluated the oxidative stress markers such as superoxide dismutase (SOD), thiobarbituric acid reactive substances (TBARS), glutathione peroxidase (GPX), before and after treatment. By comparing the mean values of SOD, TBARS in the two groups, we found that they were significantly higher in the study group compared to the control group, being higher in patients with severe disease. These values decreased after treatment, but they have remained higher than in controls. This research supports the role of oxidative stress in AMD. This paper opens the perspectives of possible elucidations of the AMD pathogenesis dilemma and supports the need to monitor the evolution by assessing the oxidative stress markers.
\end{abstract}

Keywords: age related macular degeneration, oxidative stress, superoxide dismutase, thiobarbituric acid reactive substances, glutathione peroxidase

Age-related macular degeneration is the most common cause of irreversible blindness in patients over 55 years of age [1]. AMD represents an assembly of degenerative and inflammatory lesions of the macular region, occurring in a previous normal eye. Progression of AMD is related to increased cellular stress, such as oxidative stress, inflammation and hypoxia [2]. Oxidative stress is characterized by an imbalance between the level of oxidation products and the antioxidant capacity of the body. Increased oxygen consumption, constant phagocytosis, elevated levels of polyunsaturated fatty acids and exposure to bright radiation provide an ideal environment for oxidative stress in the macular region [3]. Oxidative stress is a potent inducer of endoplasmic reticulum stress but, on the other hand, it can support oxidative stress and aggravate pathogenesis [4]. Furthermore, inflammatory responses determine the production of free oxygen radicals and molecular damage at the retinal pigment epithelium RPE [5].

The increasing incidence, the late diagnosis of the disease in advanced phases, the lack of methods to prevent the occurrence of AMD, the negative socio-economic consequences, make this condition one of the ophthalmological priorities.

The aim of the study is to evaluate the role of oxidative stress in AMD and to monitor the evolution of pre-treatmen and post-treatment oxidative stress markers.

\section{Experimental part}

Materials and methods

The study was conducted at the 2nd Ophthalmology Clinic of N. Oblu Hospital, la $0^{i}$ and is a prospective, casecontrol study. The patients enrolled in the study group (59 subjects) were diagnosed with moderate AMD and neovascular form. In order to compare the results, we also followed two control groups, one with young subjects (CG1 -40 controls) and another one with eldery subjects (CG2 -
22 controls). Inclusion criteria were: the presence of atrophic and neovascular AMD, the age between 40-86 years and the exclusion criteria: patients with systemic and ocular disorders that could increase oxidative stress, such as diabetes, inflammatory and infectious diseases, vascular occlusions. Under the Patients' Rights Act, they have signed an informed consent at the beginning of the study. The study group was monitored for an average time of 12 months. Patients were divided into 3 groups according to the Age-Related Eye Disease Study classification: mild form AMD group 1, moderate form AMD group 2 and neovascular form group 3 AMD. Only patients with moderate and severe forms were enrolled. Patients underwenttreatment with antioxidant, neurotrophic agents and intravitreal injections with corticosteroids and/or antiangiogenic factors (bevacizumab).

The evolution was followed by the evaluation of oxidative stress markers such as reactive substances of thiobarbituric acid TBARS and antioxidant enzymes superoxide dismutase SOD and glutathione peroxidase GPx. Blood samples were taken in the morning; antioxidant enzymes were dosed from blood harvested on heparin. In the study group, determinations were made at T0 - the initial time, T1 - at 3 months and T2 - at 12 months and in the control groups at T0 - the initial moment.

SOD is one of the main antioxidant systems in the retina, lowering the superoxide level. The role of SOD is to accelerate the decomposition (division) of the toxic superoxide radical produced in the oxidative processes, in hydrogen peroxide and molecular oxygen. SOD activity was determined by the xanthine-oxidase method. The Fortress method uses xanthine and xanthine-oxidase (XOD) to generate superoxide radicals. The SOD activity is then measured by the degree of inhibition of the reaction.

TBARS is an early marker of lipid peroxidation. Peroxidation of lipids leads to the formation of malondialdehyde (MDA) and 4-hydroxynonenal (4-HNE).

*email: ralucadanulescu@yahoo.com; Phone:0733792259 
MDA reacts with thiobarbituric acid, leading to the formation of TBARS, which can be quantified by the colorimetric method.

GPx catalyses the reduction of hydrogen peroxide, alkyl peroxides and peroxidic fatty acids

derived from fatty acids. The enzyme catalyses the oxidation of glutathione by hydroperoxide. Oxidated glutathione is reduced in the presence of glutathione reductase and NADPH. The absorbance at $340 \mathrm{~nm}$ is then measured.

The statistical data processing (done with the STATISTICA program) aimed to establish correlations between the changes in AMD and the results of the analysis performed. Statistical comparison was performed with the Difference tests subprogram, retaining significant differences that were above $95 \%(p<0.05)$.

\section{Results and discussions}

A group of 59 subjects with AMD was investigated, of which 29 men and 30 women, with an average age of $70.19 \pm 8.54$ (ranging from 49 to 88 years). The study comprised two control groups, one with younger (CG1) and one with elderly controls, similar to the study group (CG2). CG1 comprised 40 subjects, with an average age of $42.8 \pm 11.39$ years (ranging from 28 to 62 years) and CG2 enrolled 22 subjects aged between 50 and 80 years. Both before and after treatment, the results of the analysis are presented. We will compare the analysis results (SOD, GPX, TBARS) in the group of patients with AMD, with similar data from the two control groups.

The results of the analysis in the two control groups are presented in table 1.

We analyzed SOD, GPx, TBARS values in patients with AMD at baseline and after treatment (tables 2- 4). In the total group, SOD mean value decreases significantly from $312.1 \mathrm{U} / \mathrm{mL}$ to $297.03 \mathrm{U} / \mathrm{mL}$ in three months after treatment ( $p<0.0000001)$, also significantly decreasing one year after treatment (from $297.03 \mathrm{U} / \mathrm{mL}$ for three months at $258.98 \mathrm{U} / \mathrm{mL}, p<0.0000001$ ). The mean value of GPX increases significantly from $3202.54 \mathrm{U} / \mathrm{L}$ to $3281.08 \mathrm{U} / \mathrm{L}$ $(p=0.0019)$ three months after treatment, after which it decreases, but not statistically significant one year after treatment. The mean TBARS value decreases significantly over three months (from $4.42 \mathrm{nmol} / \mathrm{mL}$ to $4.10 \mathrm{nmol} / \mathrm{mL}$, p $<0.0000001$ ) and one year after treatment (from $4.10 \mathrm{nmol} /$ $\mathrm{mL}$ at 3 months, to $4.00 \mathrm{nmol} / \mathrm{mL}, \mathrm{p}=0.015$ ).

At the first examination, after comparing the mean values of the patients' analysis with moderate AMD and neovascular AMD, the following statistically significant differences were found: mean SOD value was significantly higher in patients with neovascular AMD $(337.78 \mathrm{U} / \mathrm{mL} \pm$ 25.49 versus $271.91 \mathrm{U} / \mathrm{mL} \pm 15.46, p<0.00001$ ), the mean value of GPx was significantly higher in patients with moderate AMD (3684.61 U/L \pm 239.8 versus $2894.56 \mathrm{U} / \mathrm{L}$ $\pm 257.87, p<0.00001$ ), the mean TBARS value was significantly higher in patients with neovascular AMD (4.72 $\mathrm{nmol} / \mathrm{mL} \pm 0.27$ versus $3.95 \mathrm{nmol} / \mathrm{mL} \pm 0.26, \mathrm{p}<$ $0.00001)$. The mean value of the analysis in the moderate AMD group does not differ significantly from that of the elderly control group.

We then evaluated the efficacy of treatment for each group (moderate AMD and neovascular AMD) according to the results of the laboratory analysis. In the moderate AMD group, the mean SOD value decreases significantly three months after treatment from $271.91 \mathrm{U} / \mathrm{mL}$ to 261.57 $\mathrm{U} / \mathrm{mL}(p=0.000004)$, one year after treatment, the change was not significant. In the neovascular AMD group, the mean SOD value was significantly reduced three months after treatment (from $337.78 \mathrm{U} / \mathrm{mL}$ to $319.69 \mathrm{U} / \mathrm{mL}, p=$ 0.000001 ) and one year after treatment (from $319.69 \mathrm{U} /$ $\mathrm{mL}$ at three months to $256.33 \mathrm{U} / \mathrm{mL}, \mathrm{p}<0.0000001$ ) (fig. 1).

We have further analysed GPx evolution according to the severity of the diagnosis. In the moderate AMD group, the mean GPx increased significantly three months after treatment from $3684.61 \mathrm{U} / \mathrm{L}$ to $3910.35 \mathrm{U} / \mathrm{L}$ ( $p$ $<0.0000001$ ), then significantly decreased one year after treatment, from $3910.35 \mathrm{U} / \mathrm{L}$ to $3762.48 \mathrm{U} / \mathrm{L}(p=0.025)$ (fig. 2).

Regarding the evolution of TBARS, in the moderate AMD group, the mean TBARS value decreases significantly three months after treatment from $3.95 \mathrm{nmol} / \mathrm{mL}$ to $3.75 \mathrm{nmol} /$ $\mathrm{mL}(p=0.0003)$, also continuing to decrease one year after

\begin{tabular}{|l|l|r|r|r|r|}
\hline \multicolumn{1}{|c|}{ Analysis } & N & Average & Minimum & Maximum & Std. Dev. \\
\hline SOD (U/ml) elderly control group CG2 & 22 & 269.55 & 248.0 & 295.0 & 13.78 \\
\hline GPx (U/1) elderly control group CG2 & 22 & 4115.64 & 3576.0 & 4573.0 & 299.80 \\
\hline TBARS (nmol/ml) elderly control group CG2 & 22 & 3.91 & 3.5 & 4.1 & 0.16 \\
\hline & & & & & \\
\hline SOD (U/ml) young control group CG1 & 40 & 201.78 & 19.0 & 311.0 & 45.54 \\
\hline GPx (U/1) young control group CG1 & 40 & 6458.38 & 4329.0 & 9658.0 & 1364.31 \\
\hline TBARS (nmol/ml) young control group CG1 & 40 & 3.15 & 2.0 & 4.3 & 0.66 \\
\hline
\end{tabular}

Table 1

INDICATORS OF MEAN VALUES OF ANALYSIS PERFORMED IN CONTROL GROUPS

\begin{tabular}{|l|c|c|c|c|c|}
\hline \multicolumn{7}{|c|}{ Analysis } & N & Average & Minimum & $\begin{array}{c}\text { Maximu } \\
\mathbf{m}\end{array}$ & Std. Dev. \\
\hline \multicolumn{7}{|c|}{ Total Group } \\
\hline SOD (U/ml) & 59 & 312.10 & 241.00 & 387.00 & 39.15 \\
\hline GPx (U/1) & 59 & 3202.54 & 2325.00 & 4032.00 & 461.49 \\
\hline TBARS (nmol/ml) & 59 & 4.42 & 3.30 & 5.59 & 0.46 \\
\hline
\end{tabular}

\begin{tabular}{|l|c|c|c|c|c|}
\hline \multicolumn{7}{|c|}{ Analysis } & N & Average & Minimum & Maximum & Std. Dev. \\
\hline SOD (U/ml) & 59 & 297.03 & 240.00 & 375.00 & 34.03 \\
\hline GPx (U/1) & 59 & 3281.08 & 2257.00 & 4327.00 & 587.91 \\
\hline TBARS (nmol/ml) & 59 & 4.10 & 3.40 & 4.86 & 0.37 \\
\hline
\end{tabular}

Table 3

RESULTS OF ANALYSIS AT 3 MONTHS AFTER TREATMENT

\begin{tabular}{|l|c|c|c|c|c|}
\hline \multicolumn{7}{|c|}{ Analysis } & N & Average & Minimum & Maximum & Std. Dev. \\
\hline \multicolumn{7}{|c|}{ Total Group } \\
\hline SOD (U/ml) & 59 & 258.98 & 125.00 & 352.00 & 58.38 \\
\hline GPx (U/1) & 59 & 3191.41 & 2121.00 & 7967.00 & 907.21 \\
\hline TBARS (nmol/ml) & 59 & 4.00 & 3.40 & 4.90 & 0.39 \\
\hline
\end{tabular}

Table 4

RESULTS OF ANALYSIS AT 12 MONTHS AFTER TREATMENT 


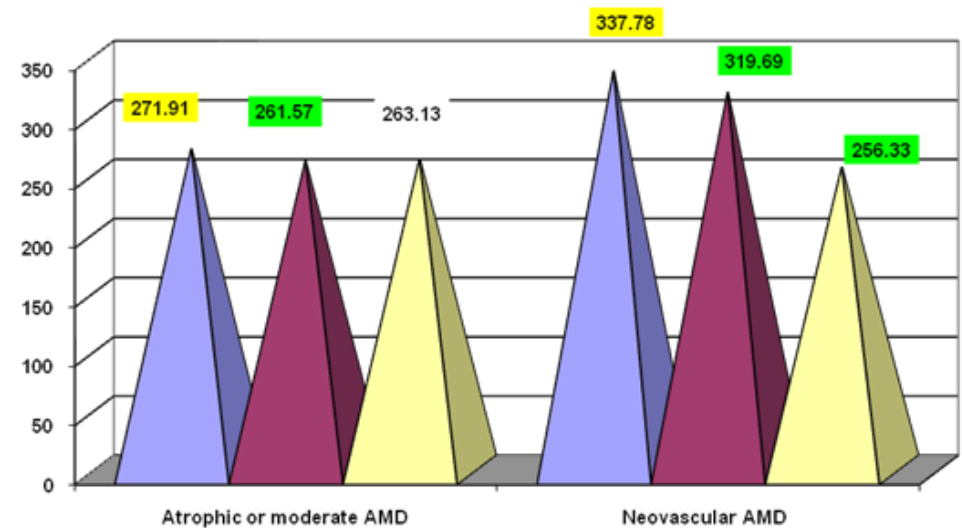

aBefore treatment a3 months after treatment Q1 vear after treatment

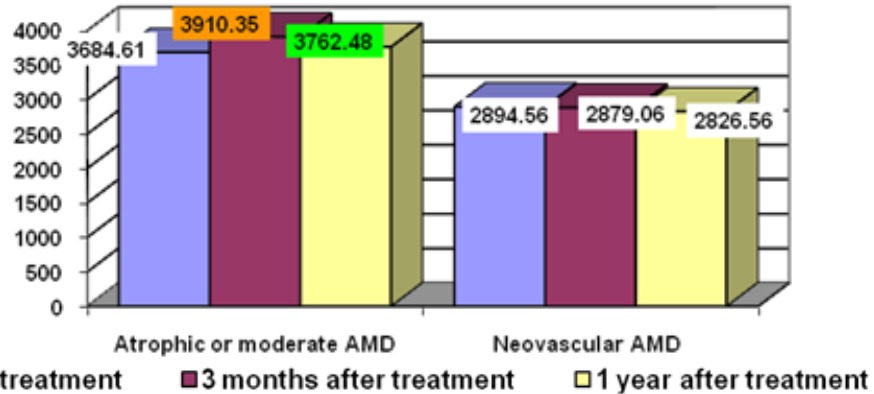

Fig. 1. Evolution of the mean value of $S O D \mathrm{U} / \mathrm{mL}$

Fig. 2. Evolution of the mean value of GPx U/L

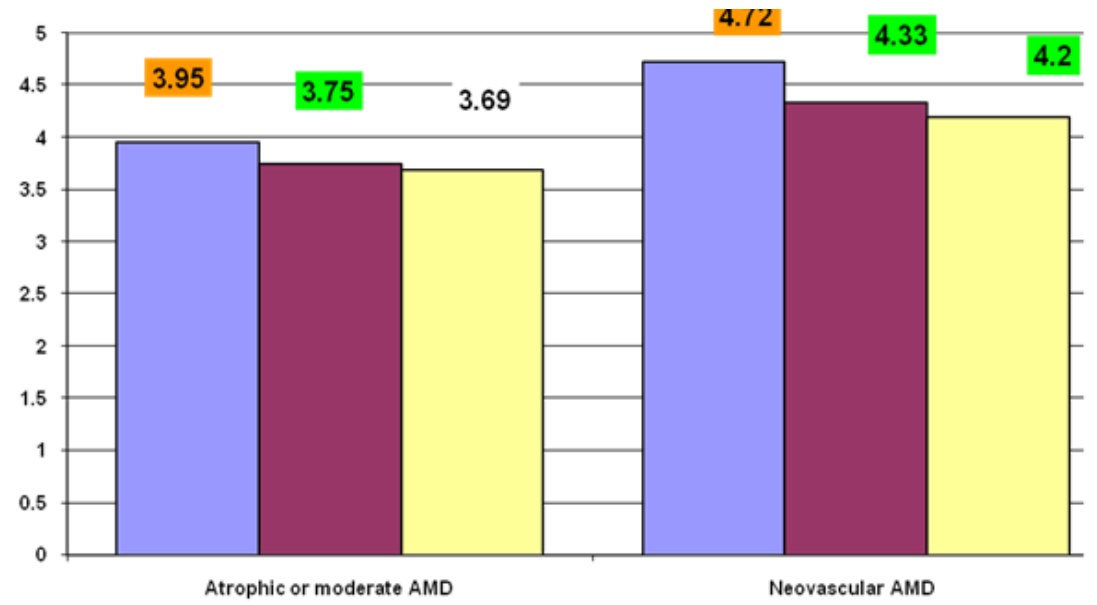

Fig. 3. Evolution of the mean value of TBARS $\mathrm{nmol} / \mathrm{mL}$

$\square$ Before treatment $\quad \square 3$ months after treatment $\quad$ 1 year

treatment, but insignificantly statistically (from $3.75 \mathrm{nmol} /$ $\mathrm{mL}$ to $3.69 \mathrm{nmol} / \mathrm{mL}$ ). In the neovascular AMD group, the mean TBARS value was significantly reduced three months after treatment (from $4.72 \mathrm{nmol} / \mathrm{mL}$ to $4.33 \mathrm{nmol} / \mathrm{mL}$, p $<.0000001$ ) and one year after treatment ( from $4.33 \mathrm{nmol} /$ $\mathrm{mL}$ at three months to $4.20 \mathrm{nmol} / \mathrm{mL}$ at one year, $p=0.03$ ) (fig. 3).

The frequencies of normal, low, elevated SOD, GPx and TBARS cases prior to treatment were compared with those of three months and one year after treatment (fig. 4).

Multiple studies have indicated that oxidative stress w ould play a crucial role in the pathogenesis of AMD. Crabb et. al. studied the drusen's composition in AMD patients and found elevated oxidized proteins [6]. J oe Hollyfield attempted to understand the composition and distribution of drusens' proteins, identifying many modified proteins and carbohydrate oxidation products $[7,8]$.

SOD is one of the main antioxidant systems in the retina, lowering the superoxide level. The enzyme grows through a feedback mechanism, along with the increase in oxidative stress, trying to keep the balance between antioxidants and free radicals.

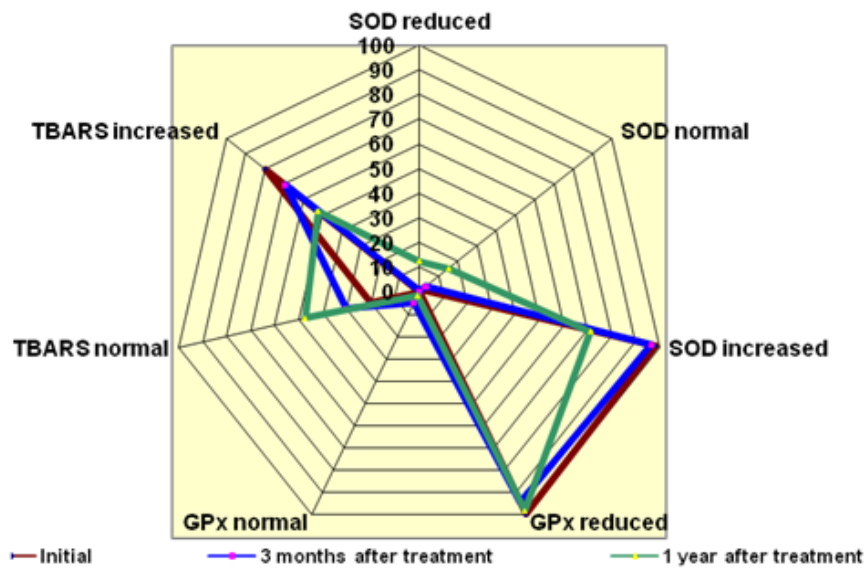

Fig. 4. Evolution of analysis results

The comparisons of the mean values of SOD in the two subgroups with AMD and the two control groups showed that at the first examination, both the AMD and the elderly groups had significantly higher SOD mean values compared to the young control group. One year after 
treatment, between the AMD patients and the elderly group, there are no significant differences.

Yukata Imamura studied the changes that occurred at retinal level in deficient mice in SOD1. They presented many key elements of human AMD, such as drusens, Bruch membrane thickening and choroidal neovascularization. RPE cells of these animals exhibited changes induced by oxidative stress, the junction integrity being compromised [9]. SOD2 (MnSOD) is located in the mitochondrial matrix and converts the superoxide anion produced by anaerobic respiration into hydrogen peroxide, an important step in cellular defense against oxidative stress. In MnSOD deficient mice Ohira et al. found morphological changes in the RPE and Bruch membrane, such as the accumulation of oxidized proteins [10]. Suppressing MnSOD expression causes increased levels of superoxide anion. Recent studies have shown that mice deficient in SOD2 exhibit retinal lesions earlier than those depleted by other antioxidant enzymes that have RPE damage only after one year.

Glutathione-peroxidase (GPx) GPxl and GPx4 protects RPE cells from the action of oxidative stress. The comparisons between GPx mean values in the two AMD subgroups with the control groups revealed at the first examination a mean value of GPX significantly lower in the AMD group compared to the young control group. Compared to the elderly control group, the mean GPx value is significantly lower in the neovascular AMD subgroup, the moderate AMD subgroup being not significantly differentiated from the elderly control group. At three months and one year after treatment, the same differences remain.

Glutathione peroxidase (GPx or GPx1) is present in the retina and is involved in antioxidant responses to light injuries [11]. GPxl activation in the external retina would be a crucial factor in response to photo-oxidative stress, preventing lipid peroxidation and photoreceptor apoptosis. Gosbell et al. have shown that mice deficient in GPxl have an increased susceptibility to oxidative stress and light aggression. GPx - deficient mice produce a model for exemplifying the role of the antioxidant enzyme pathway in intracellular regulation of oxygen free radicals [12,13]. It has been shown that deficientmice in GPx1 do notundergo changes under normal physiological conditions, but are susceptible to the action of oxidative stress. ERG studies in these patients show a decrease in the amplitude and delay of the a wave, suggesting that the photoreceptor function may be compromised. At the same time, it is interesting to note that the external segments of photoreceptors of these mice are shorter. Chronic dysfunction of antioxidant enzyme mechanisms can cause lesions that activate antioxidant enzymes, other non-enzymatic antioxidants and neuroprotective endogenous products to compensate for the lack of GPx. GPx4 is an isoenzyme acting on lipid hydroperoxides and is believed to be associated with external segments of lipid-rich photoreceptors [14]. Elevated basal levels of GPX4 may be an important factor in the protection against oxidative stress.

TBARS is one of the early markers of oxidative stress used in human and animal studies. However, this marker was less studied in relation to AMD. By comparing the values of the analysis obtained in the study group with those from the control groups, we noticed that at the first examination the group with AMD (especially neovascular AMD) has the mean TBARS value significantly higher than the young and elderly control group, whereas the mean value of TBARS in the moderate AMD group does not differ significantly from that of the elderly control group. Three months after treatment, the mean TBARS value was significantly higher in the elderly control group and remained the same at one year after treatment.

The results of the study are consistent with other studies that claim that oxidative stress plays a key role in this condition [15-19].

\section{Conclusions}

This research supports the role of oxidative stress in AMD and highlights the role of treatment against these risk factors. The mean values of SOD and TBARS in the study group were significantly higher both pre and post-treatment, consistent with the severity of the disease, compared to the values recorded in the control groups. The mean values of the analysis in the study group were higher at the first examination compared with the second examination after treatment, thus supporting its beneficial effect. GPx mean values were significantly lower both pre and post treatment. Particularly in patients with neovascular AMD, markers such as SOD, TBARS and GPx may be considered predictors of disease progression and monitoring is required. This paper opens the perspectives of possible elucidations of the AMD pathogenesis dilemma and supports the need to monitor the evolution by assessing the oxidative stress markers.

\section{References}

1. DE JONG, P., N. Engl. J. Med., 355, 2006, p. 1474-1485

2. DONG, A., XIE, B., SHEN, J., J. Cell, Physiol., 219, no. 3, 2009, p. $544-552$

3. BONNE, C., MULLER, A., J. Fr. Ophtalmol., 23, 2000, p. 835- 840

4. SALMINEN, A., KAUPPINEN, A., HYTTINEN, JM., Mol. Med., 16, no. 11-12, 2010, p. 535-542

5. JANIK-PAPIS, K., ULINSKA, M., KRZYZANOWSKA, A., Klin. Oczna., 111, 2009, p. 168-173

6. CRABB. JW., MIYAGI, M., GU, X., Proc. Natil. Acad. Sci. USA, 99, 2002, p. 14682-14687

7. HOLLYFIELD, JG., CRABB, JW., SALOMON, RG., Adv. Exp. Med. Biol., 533, 2003, p. 83-89

8. HOLLYFIELD, JG., Invest. Ophthalmol. Vil. Sci, 51, no. 3, 2010, p. 1275-1281

9. IMAMURA, Y., NODA, S., HASHIZUME, K., Proc. Natl. Acad. Sci. USA, 103, 2006, p. 11282-11287

10. KASAHARA, E., LIN, LR., HO, YS., REDDY, VN, Invest. Ophthalmol. Vis. Sci., 46, 2005, p. 3426-3434

11. OHIRA, A., TANITO, M., KAIDZU, S., KONDO, T., Invest. Ophthalmol. Vi.s Sci., 44, 2003, p. 1230-1236

12. GOSBELL, AD., STEFANOVIC, N., SCURR, LL., Invest. Ophthalmol. Vis. Sci., 47, 2006, p. 2613-2622

13. DE HAAN, JB., BLADIER, C., GRIFFITHS, P., J. Biol. Chem., 273, 1998, p. 22528-22536

14. BASKOL, G., KARAKUCUK, S., ONER, A., Erciyes. Med. J., 26, 2004, p. 7-11

15. SHEN, XL., JIA, J H., ZHAO, P., FAN, R., PAN, XY., YANG, HM., LIU, L., J. Nutr. Health. Aging., 16, no. 3, 2012 , p. 201-204

16. HOLLYFIELD, JG., BONILHA, VL., LU, L., Nat. Med., 14, 2008, p. 194-198

17. JIA, L., DONG, Y., YANG, H., Aging. Clin. Exp. Res., 23, no 4, 2011, p. 264-267

18. DONG, A., SHEN, J., KRAUSE, M., J. Cell. Physiol., 208, 2006, p. 516-526

19. LIBBY, RT., GOULD, DB., Adv. Exp. Med. Biol., 664, 2010, p. 403409.

Manuscript received: 14.05 .2018 